Received: 16.05 .2018

Revised: 17.10 .2018

Accepted: 26.10 .2018

DOI: $10.17804 / 2410-9908.2018 .6 .214-221$

\title{
THE DEVELOPMENT OF TOPOLOGICAL DIAGNOSTIC METHODS OF ASYNCHRONOUS ELECTRIC MACHINES
}

\author{
S. P. Kurilin ${ }^{\text {a)* }}$ and V. N. Denisov ${ }^{\text {b) }}$ \\ National Research University «Moscow Power Engineering Institute» in Smolensk \\ 1, Energeticheskiy Dr., Smolensk, 2014013, Russian Federation \\ a) (iD https://orcid.org/0000-0001-7843-275X kurilinsp@inbox.ru; \\ b) (iD https://orcid.org/0000-0003-3019-7855 dvalnik@mail.ru \\ ${ }^{*}$ Corresponding author. E-mail: kurilinsp@inbox.ru \\ Address for correspondence: Energeticheskiy proyezd, 1, Smolensk, 2014013, Russian Federation \\ Tel.: +7910 7128836
}

The article develops scientific foundations for the topological diagnostic methods of asynchronous electric machines (AEM) towards the development of practical applications. The topological diagnostics is based on testing the parametric characteristics of the AEM vector space or its separate subspaces. Methods for testing separate subspaces of the vector space and methods for testing AEM stators and rotors are discussed; some specialized diagnostic factors are illustrated by calculation examples. The problems stated are solved by linear algebra methods.

Keywords: topological diagnostics, diagnostic method, asynchronous electric machine, vector space.

\section{References}

1. Braun M., Rautani J., Petil D. Diagnostics and troubleshooting of electrical equipment and control circuits, Elsevier, 2005.

2. Ameid T., Menacer A., Talhaoui H., Harzelli I. Broken rotor bar fault diagnosis using fast Fourier transform applied to field-oriented control induction machine: simulation and experimental study. International Journal of Advanced Manufacturing Technology, 2017, vol. 92 (1-4), pp. 917-928. DOI: $10.1007 / \mathrm{s} 00170-017-0143-2$.

3. Bernat P., Hytka Z., Kacor P. Indication of failures of rotor bar on induction machine with squirrel cage rotor in its external electromagnetic field. In: Proceedings of the 16th International Scientific Conference on Electric Power Engineering (EPE), 2015, pp. 691-696. ISBN: 978-1-4673-6788-2.

4. Kopylov I.P. Matematicheskoe modelirovanie jelektricheskih mashin [Mathematical modeling of electrical machines]. Moscow, Vysshaya Shkola Publ., 2001, 327 p. (In Russian).

5. Ivanov-Smolenskiy A.V. Electrical machines, vol. 2. Mir Publishers.

6. Kurilin S.P., Denisov V.N. Metody i prilozheniya matematicheskogo modelirovaniya $v$ elektrotechnike [Methods and Applications of Mathematical Modeling in Electrical Engineering: Monograph]. Smolensk, Smolenskiy Filial RUK Publ, 2014, 242 p. ISBN 978-5-91805-037-8. (In Russian).

7. Nos O.V. Mathematical model of the asynchronous engine in the linear spaces connected to the stator and a rotor. Izvestiya Vysshikh Uchebnykh Zavedenii, 2008, no. 2, pp. 14-20. (In Russian).

8. Denisov V.N., Kurilin S.P. Method of diagnosing phase-rotor motors. RF Patent 2392632, 2010. (In Russian). 
Подана в журнал: 16.05.2018

УДК 621.313.333:(512+514.1+515.1)

DOI: $10.17804 / 2410-9908.2018 .6 .214-221$

\title{
РАЗРАБОТКА МЕТОДОВ ТОПОЛОГИЧЕСКОЙ ДИАГНОСТИКИ АСИНХРОННЫХ ЭЛЕКТРИЧЕСКИХ МАШИН
}

\author{
С.П. Курилин ${ }^{\text {a* }}$, В.Н. Денисов ${ }^{\text {б) }}$ \\ Филиал ФГБОУ ВО «НИУ «МЭИ» в г. Смоленске, \\ Энергетический пр. 1, 214013, Смоленск, Российская Федераџия \\ a) (ID https://orcid.org/0000-0001-7843-275X $ه$ kurilinsp@inbox.ru; \\ б) (iD https://orcid.org/0000-0003-3019-7855 dvalnik@mail.ru \\ *Ответственный автор. Электронная почта: kurilinsp@inbox.ru \\ Адрес для переписки: Энергетический пр. 1, г. Смоленск, 214013, Российская Федерация \\ Тел.: +7-910-712-88-36
}

В статье развиваются научные основы методов топологической диагностики асинхронных электрических машин (АЭМ) в направлении разработки практических приложений. Топологическая диагностика базируется на тестировании параметрических свойств векторного пространства АЭМ или его отдельных подпространств. В статье обсуждаются методы тестирования отдельных подпространств векторного пространства, методы тестирования статора и ротора АЭМ, а также на расчетных примерах представлены некоторые из специализированных диагностических факторов. Поставленные задачи решаются методами линейной алгебры.

Ключевые слова: топологическая диагностика, метод диагностики, асинхронная электрическая машина, векторное пространство.

\section{1. Введение}

Проблема повышения эксплуатационной надежности асинхронных электрических машин (АЭМ) решается организацией системы их периодической эксплуатационной диагностики. Значение эксплуатационной диагностики АЭМ для поддержания рабочего состояния парка технологического электрооборудования весьма высоко. Это вызвано тем, что потери от внезапной остановки технологического оборудования многократно превышают потери от плановой замены электродвигателя в его составе. В связи с этим интерес специалистов и ученых к вопросам эксплуатационной диагностики в настоящее время высок $[1,2]$.

Существующие методы эксплуатационной диагностики АЭМ $[1,3]$ основаны на фиксации и анализе внешних потоков энергии электрической машины: интенсивности электромагнитного, теплового или акустического поля, величин потребляемых токов или момента на ее валу. Теоретической базой методов диагностики длительное время служила классическая теория электрической машины, в частности ее математическая концепция $[4,5]$. В настоящее время формируется топологическая концепция АЭМ [6, 7], обосновывающая принципиально новые - топологические - методы эксплуатационной диагностики.

Топологическая диагностика базируется на тестировании параметрических свойств векторного пространства АЭМ или его отдельных подпространств [6]. Размерность векторного пространства АЭМ равна большему числу фаз одной из ее обмоток. Как правило, тестирование проводится через обмотку статора. При этом тестируется трехмерное подпространство векторного пространства, которое является общим для обмоток статора и ротора. Представление об этом подпространстве для случая однородной (неповрежденной) АЭМ дает рисунок [6]. 


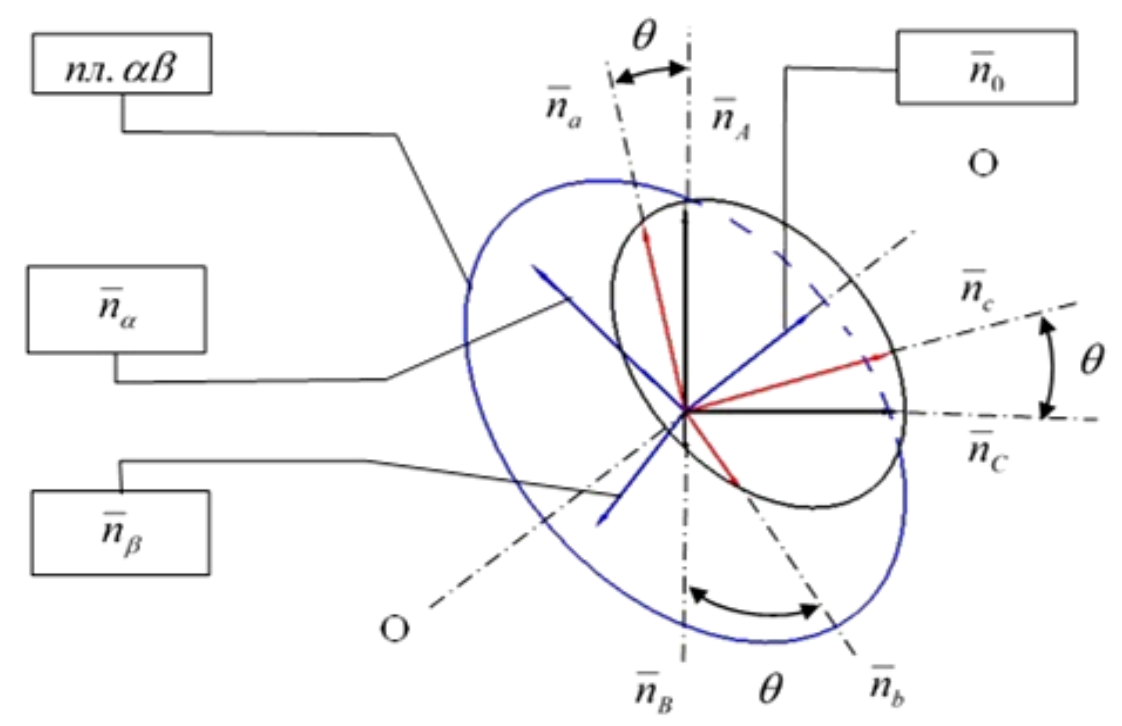

Базисы и подпространства векторного пространства однородной АЭМ

На рисунке представлены ортонормированные фазные базисы обмотки статора $\left\{\boldsymbol{n}_{A}, \boldsymbol{n}_{B}, \boldsymbol{n}_{C}\right\}$

$$
\boldsymbol{n}_{A}=\left(\begin{array}{l}
1 \\
0 \\
0
\end{array}\right), \boldsymbol{n}_{B}=\left(\begin{array}{l}
0 \\
1 \\
0
\end{array}\right), \boldsymbol{n}_{C}=\left(\begin{array}{l}
0 \\
0 \\
1
\end{array}\right)
$$

и обмотки ротора $\left\{\boldsymbol{n}_{a}, \boldsymbol{n}_{b}, \boldsymbol{n}_{c}\right\}$.

$$
\boldsymbol{n}_{a}=\left(\begin{array}{l}
1 \\
0 \\
0
\end{array}\right), \boldsymbol{n}_{b}=\left(\begin{array}{l}
0 \\
1 \\
0
\end{array}\right), \boldsymbol{n}_{c}=\left(\begin{array}{l}
0 \\
0 \\
1
\end{array}\right) .
$$

Базис обмотки ротора повернут относительно базиса обмотки статора на угол и вокруг оси ОО, заданной направляющим вектором $\boldsymbol{n}_{0}$. На рисунке представлена плоскость $\alpha \beta$ и параллельная ей плоскость, на которой находятся концы векторов $\boldsymbol{n}_{a}, \boldsymbol{n}_{b}, \boldsymbol{n}_{c}$ при вращении ротора,атакже один из канонических базисов векторного пространства однородной АЭМ $\left\{\boldsymbol{n}_{0}, \boldsymbol{n}_{\alpha}, \boldsymbol{n}_{\beta}\right\}$

$$
\boldsymbol{n}_{\alpha}=\sqrt{\frac{2}{3}}\left(\begin{array}{c}
-\frac{1}{2} \\
1 \\
-\frac{1}{2}
\end{array}\right), \boldsymbol{n}_{\beta}=\sqrt{\frac{2}{3}}\left(\begin{array}{c}
-\frac{\sqrt{3}}{2} \\
0 \\
\frac{\sqrt{3}}{2}
\end{array}\right), \boldsymbol{n}_{0}=\frac{1}{\sqrt{3}}\left(\begin{array}{l}
1 \\
1 \\
1
\end{array}\right) .
$$

Этот базис выделяет в векторном пространстве АЭМ два подпространства: двумерную плоскость $\alpha \beta$ и одномерное подпространство нулевой последовательности 0. Подпространство $\alpha \beta$ является рабочей областью векторного пространства неповрежденной машины. 
В поврежденной АЭМ векторы токов, напряжений и ЭДС могут располагаться в любой области векторного пространства.

\section{2. Постановка задачи и методы решения}

Начиная исследование, авторы ставили следующие основные задачи.

1. Сформировать методологию топологической диагностики, позволяющую получить достоверные данные для заключения о техническом состоянии и остаточном ресурсе АЭМ.

2. Сформировать информационно-математический аппарат, который с необходимой полнотой и детальностью отображает текущее состояние векторного пространства АЭМ и его изменения во времени.

При этом предполагалось, что на базе топологического подхода могут быть сформированы различные программы диагностических испытаний АЭМ, в ходе которых состав диагностических факторов может быть дополнен или скорректирован, а принцип и методы диагностических испытаний станут основой разработки технических средств диагностики АЭМ.

В соответствии с методологией топологической диагностики, при проведении диагностического обследования одной из координат вектора напряжения обмотки статора придают вид дельта-функции Дирака $\delta(t)$. Пусть, например, импульс напряжения в форме дельтафункции подается на фазу $A$ обмотки статора, а фазы $B$ и $C$ статора и фазы ротора замкнуты накоротко

$$
\left(\begin{array}{c}
\delta(t) \\
0 \\
0
\end{array}\right)
$$

тогда реакцией АЭМ на воздействие будет вектор-столбец, составленный из функций Грина

$$
\left(\begin{array}{l}
g_{A A}(t) \\
g_{B A}(t) \\
g_{C A}(t)
\end{array}\right)
$$

Аналогичные векторы-столбцы получаются и при подаче дельта-функции на фазы $B$ иС обмотки статора. Совокупность этих реакций образует матрицу Грина - токовую реакцию АЭМ на воздействия в форме дельта-функций:

$$
G(t)=\left(\begin{array}{lll}
g_{A A}(t) & g_{A B}(t) & g_{A C}(t) \\
g_{B A}(t) & g_{B B}(t) & g_{B C}(t) \\
g_{C A}(t) & g_{C B}(t) & g_{C C}(t)
\end{array}\right)
$$

Элементами матрицы Грина являются собственные и взаимные электрические проводимости фаз статора при короткозамкнутом роторе. Эти элементы используются в качестве диагностических факторов и хранятся в базе данных с момента проведения выходной диагностики до конца срока эксплуатации АЭМ.

Заключение по результатам диагностического обследования делается на основе сравнения его результатов с данными эталонного состояния. Матрица Грина для эталонного состояния формируется при выходных испытаниях АЭМ на заводе-изготовителе или на ремонтном предприятии. Оценка однородности матрицы Грина дает основания для заключения о наличии повреждений в структуре объекта, а наличие отклонений от эталонной матрицы свидетельствует об эксплуатационном старении объекта. При этом скорость эксплуатационного старения позволяет оценить остаточный ресурс АЭМ. 


\section{3. Результаты и обсуждение}

Выше приведена методика тестирования векторного пространства по осям базиса $\left\{\boldsymbol{n}_{A}, \boldsymbol{n}_{B}, \boldsymbol{n}_{C}\right\}$ Получаемые при этом результаты отражают общую однородность или неоднородность векторного пространства АЭМ. Результаты тестирования представляют собой усредненные электрические проводимости векторного пространства по осям базиса с учетом их различий в подпространствах $\alpha \beta$ и 0 . По направленности данная методика ориентирована на определение степени эксплуатационного износа и остаточного ресурса объекта.

Вместе с тем задачи эксплуатационной диагностики могут быть весьма многообразны. В ряде случаев может потребоваться детальное тестирование отдельных подпространств векторного пространства. В первую очередь это относится к рабочей области векторного пространства АЭМ - подпространству $\alpha \beta$. В других ситуациях, в случаях уточнения места повреждения или ускоренного старения, оказываются необходимыми тестирования отдельных фрагментов активной части АЭМ - статора и ротора.

Кроме того, при разработке методов диагностического обследования одной из ключевых задач является поиск диагностических факторов, адекватных поставленным задачам. При реализации различных программ диагностических испытаний в состав диагностических факторов кроме матрицы Грина могут быть включены и дополнительные специализированные факторы.

Далее авторы намерены обсудить методы тестирования отдельных подпространств векторного пространства, методы тестирования статора и ротора АЭМ, а также представить некоторые из специализированных диагностических факторов.

\section{1. Тестирование отдельных подпространств векторного пространства}

При тестировании подпространства $\alpha \beta$ импульсные сигналы подаются по различным осям плоскости $\alpha \beta$, а фазы статора не подключенные к источнику импульсного напряжения замыкаются накоротко. Во всех случаях, кроме тестирования, обмотки статора, испытания проводятся при неподвижном роторе.

Таким образом, по результатам трех испытаний векторами импульсного напряжения

$$
\left(\begin{array}{c}
\delta(t) \\
-\delta(t) \\
0
\end{array}\right),\left(\begin{array}{c}
0 \\
\delta(t) \\
-\delta(t)
\end{array}\right),\left(\begin{array}{c}
-\delta(t) \\
0 \\
\delta(t)
\end{array}\right),
$$

формируется матрица Грина с электрическими проводимостями плоскости $\alpha \beta$ векторного пространства

$$
G(t)=\left(\begin{array}{lll}
g_{A A}(t) & g_{A B}(t) & g_{A C}(t) \\
g_{B A}(t) & g_{B B}(t) & g_{B C}(t) \\
g_{C A}(t) & g_{C B}(t) & g_{C C}(t)
\end{array}\right)
$$

Здесь символами $A, B, C$ обозначены оси плоскости $\alpha \beta$, заданные проекциями векторов $\boldsymbol{n}_{A}, \boldsymbol{n}_{B}, \boldsymbol{n}_{C}$ на эту плоскость (рисунок). Соответственно и элементы матрицы Грина, в данном случае, представляют собой собственные и взаимные электрические проводимости эквивалентных фаз статора, расположенных в плоскости $\alpha \beta$ по этим осям.

При тестировании подпространства 0 импульсный сигнал подается на фазы обмотки статора, соединенные в схему «разомкнутый треугольник». В результате испытания появля- 
ется функция Грина для токов нулевой последовательности $g_{0}(t)$, представляющая собой электрическую проводимость векторного пространства по оси ОО (рис. 1).

\section{2. Тестирование статора и ротора АЭМ}

Тестирование статора АЭМ должно производиться при обесточенной обмотке ротора. Для АЭМ с короткозамкнутым ротором, в рамках эксплуатационной диагностики, довести ток обмотки ротора до нуля невозможно. По этой причине испытания следует провести в режиме холостого хода, в котором ток ротора приблизительно равен нулю. Тестовые испытания могут быть проведены по методике в соответствии с материалом разд. 3.1.

При асинхронно вращающемся роторе матрица Грина не будет симметричуой, но будет обладать симметрией следующего вида

$$
G(t)=\left(\begin{array}{lll}
a(t) & b(t) & c(t) \\
c(t) & a(t) & b(t) \\
b(t) & c(t) & a(t)
\end{array}\right)
$$

Аналогичную структуру должна иметь и эталонная матрица.

Тестирование короткозамкнутого ротора АЭМ производиться по обычной методике (см. выражение (1)). При этом испытания повторяются для различных угловых положений ротора

$$
\theta=\theta_{i}=\frac{2 \pi p}{z} i, i=0,1,2, \ldots
$$

где $p$ - число пар полюсов АЭМ; $z$ - число фаз ротора АЭМ.

В результате формируется матричная функция Грина

$$
G(t, \theta)=\left(\begin{array}{lll}
g_{A A}(t, \theta) & g_{A B}(t, \theta) & g_{A C}(t, \theta) \\
g_{B A}(t, \theta) & g_{B B}(t, \theta) & g_{B C}(t, \theta) \\
g_{C A}(t, \theta) & g_{C B}(t, \theta) & g_{C C}(t, \theta)
\end{array}\right) .
$$

Независимость элементов матрицы Грина от угла и свидетельствует о параметрической и физической однородности ротора. Слабая зависимость элементов матрицы Грина от угла и свидетельствует о наличии неоднородностей ротора, а явно выраженная зависимость элементов матрицы Грина от угла и свидетельствует о наличии повреждений ротора.

\section{3. Специализированные диагностические факторы}

Далее, по результатам [6] приводятся два расчетных примера процедуры подбора диагностических факторов для случаев повреждения обмоток статора и короткозамкнутого ротора асинхронного электродвигателя. Расчеты серийного электродвигателя 4A160S4У3 мощностью 15 КВт с частотой вращения 1500 об./мин проводились на математических моделях по существующим методикам.

Пример 1. При моделировании повреждения обмотки статора оценивалась ситуация повышения сопротивления одной из фаз на 10 \% по отношению к номинальному значению. Сравнивались уровни мощности рассеяния обмотки для случаев неповрежденной и поврежденной обмотки. Полученные расчетные данные свидетельствуют о следующем.

Параметрически однородная (неповрежденная) обмотка не возбуждает междуфазный энергообмен. Определенные в расчете величины потерь активной $(908,9$ Вт) и реактивной 
(1740,4 ВАр) мощности следует считать неизбежными и соответствующими реализованному в двигателе 4A160S4У3 уровню технологии и культуры производства.

Параметрически неоднородная (поврежденная) обмотка имеет не только повышенные уровни рассеяния активной $(939,2$ Вт) и реактивной $(1798,4$ ВАр) мощности, но и возбуждает междуфазный энергообмен. Мерой интенсивности энергообмена является угол между векторами напряжения и тока в векторном пространстве, равный 0,046 радиан. Обмен, совокупной мощностью в 92,6 ВА, идет в формах активной $(42,85$ Вт) и реактивной $(82,04 \mathrm{BAp})$ мощности. Мощности обмена избыточны, так как являются следствием параметрической неоднородности векторного пространства объекта и даже косвенно не связаны с электромеханическим преобразованием энергии в нем.

В качестве специализированного диагностического фактора, указывающего на повреждение обмотки статора, в данном случае может использоваться пространственный угол между векторами напряжения и тока.

Пример 2. Моделирование повреждения короткозамкнутой клетки ротора. В расчете сравнивались токи стержней поврежденной (неоднородной) и неповрежденной (однородной) короткозамкнутой клетки ротора электродвигателя 4A160S4У3, имеющей 38 фаз. Поврежденная клетка имела удвоенное активное сопротивление одной из фаз с условным номером 19.

В результате расчета свидетельствуют, что у поврежденной клетки наблюдается резкая неоднородность векторного пространства, в частности существенное различие амплитуд фазных токов. В окрестностях точки повреждения различие достигает 2,5 номинальных значений. При этом среднее значение фазного тока клетки повышается на $16 \%$ по сравнению с неповрежденной клеткой. В данном случае специализированными диагностическими факторами, указывающими на повреждение ротора, могут быть величина потребляемого обмоткой статора тока и его пульсирующий характер.

\section{4. Заключение}

В исследовании на основе топологической теории развиваются теоретические и методологические аспекты эксплуатационной диагностики АЭМ. При этом тестирование векторных подпространств АЭМ производится импульсными функциями, а основным диагностическим фактором служит матрица Грина.

Методы топологической диагностики, разрабатываемые на платформе топологической теории, позволяют получить достоверные данные для заключения о техническом состоянии и остаточном ресурсе АЭМ. Они обладают гибкостью в отношении конкретных задач диагностического обследования и состава диагностических факторов. Кроме того, разрабатываемые методы обладают технической реализуемостью и новизной, о чем, в частности, свидетельствует [8].

Принятый в исследовании аппарат функций Грина с необходимой полнотой и детальностью отображает текущее состояние векторного пространства АЭМ и его изменения во времени. Матрица Грина является достаточно универсальным и емким диагностическим фактором для осуществления эксплуатационной диагностики. Это относится как к тестированию отдельных подпространств векторного пространства, так и к тестированию обмоток статора и ротора АЭМ по отдельности.

На базе топологического подхода могут быть сформированы различные программы диагностических испытаний АЭМ, в ходе которых состав диагностических факторов может быть дополнен или скорректирован. Матрица Грина в ряде ситуаций может быть дополнена специализированными диагностическими факторами.

Разработанные на базе теоретических и методологических положений методики и технические средства могут стать основой повышения эксплуатационной надежности асинхронных электрических машин. 


\section{Литература}

1. Браун М., Раутани Дж., Пэтил Д. Диагностика и поиск неисправностей электрооборудования и цепей управления. - М. : Издательский дом «Додэка XXI», 2007. - 328 с.

2. Broken rotor bar fault diagnosis using fast Fourier transform applied to field-oriented control induction machine: simulation and experimental study / T. Ameid, A. Menacer, H. Talhaoui, I. Harzelli // International Journal of Advanced Manufacturing Technology. - 2017. - Vol. 92 (1-4). P. 917-928. - DOI: 10.1007/s00170-017-0143-2.

3. Bernat P., Hytka Z., Kacor P. Indication of failures of rotor bar on induction machine with squirrel cage rotor in its external electromagnetic field // Proceedings of the 2015 16th international scientific conference on electric power engineering (EPE). - 2015. - P. 691-696. - ISBN: 978-14673-6788-2.

4. Копылов И. П. Математическое моделирование электрических машин. - М. : Высшая школа, 2001. -327 с.

5. Иванов-Смоленский А. В. Электрические машины. - М. : Энергия, 1980. - 928 с.

6. Курилин С. П., Денисов В. Н. Методы и приложения математического моделирования в электротехнике : монография. - Смоленск : Смоленский филиал РУК, 2014. - 242 с. ISBN 978-5-91805-037-8.

7. Нос О. В. Математическая модель асинхронного двигателя в линейных пространствах, связанных со статором и ротором // Изв. вузов. Электромеханика. - 2008. - № 2. C. $14-20$.

8. Способ диагностики электрических двигателей с фазным ротором : пат. 2392632 Рос. Федерация / Денисов В. Н., Курилин С. П. - № 2008148774/28 ; заявл. 10.12.08 ; опубл. 20.06.2010, Бюл. № 17. - 5 c. 